\title{
A Quadratic Formula for Finding the Root of an Equation
}

\author{
By Ll. G. Chambers
}

\begin{abstract}
An extension is made to Reguli Falsi involving a parabolic approximation.
\end{abstract} Formulae are derived which have convergence exponents $2,2.414,2.732$, respectively.

1. Introduction. Let $(a, A),(b, B),(c, C)$ be three points on the curve $y=f(x)$.

The parabola passing through these three points whose axis is parallel to the $x$-axis is given by

$$
x=a \frac{(y-B)(y-C)}{(A-B)(A-C)}+b \frac{(y-C)(y-A)}{(B-C)(B-A)}+c \frac{(y-A)(y-B)}{(C-A)(C-B)} .
$$

The value of $x$, denoted by $d$, at which $y$ vanishes is given by

$$
d=\frac{a B C}{(A-B)(A-C)}+\frac{b C A}{(B-C)(B-A)}+\frac{c A B}{(C-A)(C-B)} .
$$

Suppose that $\xi$ is a root of the equation $f(x)=0$ and suppose that $a=\xi+\alpha, b=$ $\xi+\beta, c=\xi+\gamma$ are approximations to the root. Then, if $d=\xi+\delta$, it can easily be seen that

$$
\begin{aligned}
\delta & =\frac{\alpha B C}{(A-B)(A-C)}+\frac{\beta C A}{(B-C)(B-A)}+\frac{\gamma A B}{(C-A)(C-B)} \\
& =\alpha \beta \gamma\left(P-Q+P^{2}\right)\{1+\text { terms of higher order }\},
\end{aligned}
$$

where $P=f^{\prime \prime}(\xi) / 2 f^{\prime}(\xi), Q=f^{\prime \prime \prime}(\xi) / 6 f^{\prime}(\xi)$. Thus

$$
\delta \doteq K \alpha \beta \gamma, \text { where } K \text { is a constant. }
$$

Many iterative formulae have been obtained for the solution of the equation $f(x)=0$, and these are readily available [2], [3]. It is the purpose of this note to indicate certain new iterative formulae which can be derived using Eq. (1.2). The associated order of convergence will be given by Eq. (1.4). Obviously the method will not be applicable for a double root, as in this case, the associated curve touches the $x$-axis and lies only on one side of it and cannot be approximated by a representation of $x$ as a polynomial in $y$. For the same reason, it is clear that it is necessary for the root to be isolated.

\section{Iterative Formulae.}

(a) Third Order Iteration. The obvious iterative sequence to consider in connec-

Received December 15, 1969, revised February 10, 1970.

AMS 1970 subject classifications. Primary 65H05; Secondary 65B99.

Key words and phrases. Numerical analysis, approximation to the root of an equation.

Copyright @ 1971, American Mathematical Society 
tion with formula (1.2) is the third-order iteration defined by $a=x_{n-2}, b=x_{n-1}, c=$ $x_{n}, d=x_{n+1}$ :

$$
\begin{aligned}
x_{n+1}= & \frac{x_{n-2} y_{n-1} y_{n}}{\left(y_{n-2}-y_{n-1}\right)\left(y_{n-2}-y_{n}\right)}+\frac{x_{n-1} y_{n-2} y_{n}}{\left(y_{n-1}-y_{n}\right)\left(y_{n-1}-y_{n-2}\right)} \\
& +\frac{x_{n} y_{n-2} y_{n-1}}{\left(y_{n}-y_{n-1}\right)\left(y_{n}-y_{n-2}\right)} .
\end{aligned}
$$

If $x_{n}=\xi+\epsilon_{n}$, then from Eq. (1.4) the convergence is determined by

$$
\epsilon_{n+1} \doteq K \epsilon_{n} \epsilon_{n-1} \epsilon_{n-2} \text {. }
$$

This is the same relation as that of Muller's method [1] and so

$$
\epsilon_{n+1} \doteq L \epsilon_{n}^{1.84}
$$

where $L$ is a constant.

(b) Improved Second Order Iterations. It does not seem to have been noticed previously that it is possible to use modifications of second-order iterations which in fact have a better convergence exponent than the third-order iteration discussed already. In these, one of the interpolation points is a function of the other two. That is: $a=x_{n-1}, b=x_{n}, d=x_{n+1}$ and $c=x_{n}^{*}$, where $x_{n}^{*}$ is some function of $x_{n-1}$ and $x_{n}$.

The iterative sequence becomes

$$
\begin{aligned}
x_{n+1}= & \frac{x_{n}^{*} y_{n-1} y_{n}}{\left(y_{n}^{*}-y_{n}\right)\left(y_{n}^{*}-y_{n-1}\right)}+\frac{x_{n-1} y_{n}^{*} y_{n}}{\left(y_{n-1}-y_{n}\right)\left(y_{n-1}-y_{n}^{*}\right)} \\
& +\frac{x_{n} y_{n}^{*} y_{n-1}}{\left(y_{n}-y_{n-1}\right)\left(y_{n}-y_{n}^{*}\right)}
\end{aligned}
$$

and the convergence is given by

$$
\epsilon_{n+1} \doteq K \epsilon_{n} \epsilon_{n-1} \epsilon_{n}^{*}
$$

with an obvious notation.

Only a few out of the many possible definitions for $x_{*}^{*}$ will be discussed here. First, the "mean" iteration will be considered. In this

$$
x_{n}^{*}=\frac{1}{2}\left(x_{n}+x_{n-1}\right),
$$

giving

$$
\epsilon_{n+1} \doteq K \epsilon_{n} \epsilon_{n-1} \cdot \frac{1}{2}\left(\epsilon_{n}+\epsilon_{n-1}\right) \doteq L \epsilon_{n} \epsilon_{n-1}^{2},
$$

as $\epsilon_{n}$ is negligible compared with $\epsilon_{n-1}$. The convergence exponent may easily be determined. Let

$$
\epsilon_{n+1}=M \epsilon_{n}^{\mu}
$$

It follows that

$$
\mu=1+\frac{2}{\mu},
$$

the appropriate root of which is $\mu=2$. Thus

$$
\epsilon_{n+1} \doteq M \epsilon_{n}^{2}
$$


and the convergence is quadratic. It is fairly easy to see that the same would be true for any linear iteration of the Wegstein type $x_{n}^{*}=g x_{n}+(1-g) x_{n-1}, g \neq 1$. If $g=1$, $x_{n}^{*}=x_{n}$ and the formula (2.4) becomes

$$
\begin{aligned}
& x_{n+1}=\frac{x_{n-1} y_{n}^{2}}{\left(y_{n-1}-y_{n}\right)^{2}}+\underset{x_{n}^{*} \rightarrow x_{n}}{\operatorname{lt}}\left\{\frac{1}{\left(y_{n}^{*}-y_{n}\right)}\left[\frac{x_{n}^{*} y_{n-1} y_{n}}{y_{n}^{*}-y_{n-1}}-\frac{x_{n} y_{n-1} y_{n}^{*}}{y_{n}-y_{n-1}}\right]\right\} \\
& \left.=\frac{x_{n-1} y_{n}^{2}}{\left(y_{n-1}-y_{n}\right)^{2}}+\left(\frac{y_{n}}{y_{n}-y_{n-1}}\right)_{x_{n}^{*} \rightarrow x_{n}} 1 \frac{x_{n}^{*} y_{n}-x_{n} y_{n}^{*}}{y_{n}^{*}-y_{n}}\right) \\
& =\frac{x_{n-1} y_{n}^{2}}{\left(y_{n-1}-y_{n}\right)^{2}}+\left(\frac{y_{n}}{y_{n}-y_{n-1}}\right)\left(\frac{y_{n}-x_{n} y_{n}^{\prime}}{y_{n}^{\prime}}\right) \text {. }
\end{aligned}
$$

This, in effect, is equivalent to taking a parabola passing through $\left(x_{n-1}, y_{n-1}\right)$ and touching the curve $y=f(x)$ at $\left(x_{n}, y_{n}\right)$. The convergence exponent is given by

$$
\epsilon_{n+1} \doteq K \epsilon_{n}^{2} \epsilon_{n-1} \text {, }
$$

because $\epsilon_{n}^{*}=\epsilon_{n}$ and if $\epsilon_{n+1}=L \epsilon_{n}^{\mu}$, then $\mu=2+1 / \mu$, and $\mu=1+\sqrt{ } 2$ and so,

$$
\epsilon_{n+1} \doteq K \epsilon_{n}^{2.414} \text {. }
$$

This procedure has the same order of convergence as a similar one discussed by Ostrowski [2]. that is

Finally, suppose that $x_{n}^{*}$ is determined by Reguli Falsi [1] as applied to $x_{n-1}, x_{n}$;

$$
x_{n}^{*}=\frac{x_{n-1} y_{n}-x_{n} y_{n-1}}{y_{n}-y_{n-1}} .
$$

$\epsilon_{n}^{*}$ is defined by an expression of the form

$$
\epsilon_{n}^{*} \doteq L \epsilon_{n} \epsilon_{n-1},
$$

and so

$$
\epsilon_{n+1} \doteq K \epsilon_{n} \epsilon_{n-1} L \epsilon_{n} \epsilon_{n-1} \doteq M \epsilon_{n}^{2} \epsilon_{n-1}^{2} .
$$

Following the usual procedure $\epsilon_{n+1}^{\mu}=N \epsilon_{n}, \mu=2+2 / \mu$ and $\mu=1+\sqrt{ } 3$,

$$
\epsilon_{n+1}^{\mu} \doteq N \epsilon_{n}^{2.732} \text {. }
$$

It is interesting to note that these improved second-order iterations all give higher convergence exponents than does the third-order iteration.

Mathematics Department

University College of North Wales

Bangor, Caernarfonshire

Wales

1. C. E. FRöBERG, Introduction to Numerical Analysis, Addison-Wesley, Reading, Mass., 1965, p. 20. MR 30 \#5457.

2. A. OstrowSKI, Solution of Equations and Systems of Equations, Pure and Appl. Math., vol. 9, Academic Press, New York, 1960, p. 84. MR 23 \#B571.

3. J. F. TRAUB, Iterative Methods for the Solution of Equations, Prentice-Hall Series in Automatic Computation, Prentice-Hall, Englewood Cliffs, N. J., 1964, p. 105. MR 29 \#6607. 